\author{
Military Technical College \\ Kobry El-Kobbah, \\ Cairo, Egypt.
}

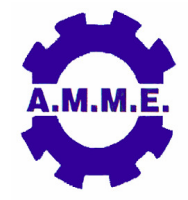

\title{
PROCEDURES FOR MATHEMATICAL MODELING OF SMALL UNMANNED AERIAL VEHICLES
}

\author{
A. S. Elharouny ${ }^{1}$, A. M. Youssef ${ }^{2}$, M. Y. Zakaria ${ }^{3}$ and M. M. Abdel-Hameed ${ }^{4}$
}

\begin{abstract}
In the past few years, the unmanned air vehicle (UAV) has found diverse applications for both civil and military missions. To achieve the stated mission, the vehicle needs to have a flight control system. For designing the control system, the flight control engineers must first develop a dynamic aircraft model and verify its accuracy. Dynamic characteristics of an aircraft are normally described in terms of its stability and control derivative values. These values are determined either experimentally; using flight tests or wind tunnel, or mathematically using computational fluid dynamics (CFD). The experimental approach is generally very accurate, but time consuming and expensive. The CFD method takes lots of CPU time and provides less precision than the wind tunnel testing. In this paper, USAF Digital DATCOM computer program is used to provide estimation of aerodynamic stability and control characteristics. Even though DATCOM provides less precision than CFD and wind tunnel tests, it works much more efficiently by reducing considerable computing time and design cost. Stability and control coefficients and derivatives obtained from DATCOM can be used to calculate the coefficients of state-space matrices. The resulting linear model can then be used for control design tasks. This paper discusses the step by step procedure for obtaining mathematical models for small UAVs. This procedure is then applied for modeling a remote controlled UAV called "Sky Raider Mach 1".
\end{abstract}

\section{KEY WORDS}

UAV, DATCOM, Modeling, PROFILI

\footnotetext{
${ }^{1}$ M. Sc. Student, Dept. of Mechatronics, Faulty of Eng., Ain Shams Univ., Cairo, Egypt.

${ }^{2}$ Egyptian Armed Forces.

${ }^{3}$ Egyptian Armed Forces

${ }^{4}$ Professor, Dept. of Mechatronics, Faulty of Eng., Ain Shams Univ., Cairo, Egypt.
} 


\section{INTRODUCTION}

In the past few years, the UAV has found diverse applications for both civil and military missions. To achieve the stated mission, the vehicle needs to have a flight control system. Having an accurate mathematical model of the airplane is an essential prerequisite for control system design. Dynamic characteristics of an aircraft are normally described in terms of its stability and control derivative values. Typically, these coefficients are experimentally determined using flight tests or wind tunnel, or mathematically using CFD. The most accurate method involves flight testing the actual aircraft, unfortunately these tests are very expensive, sometimes risky since they require the operational aircraft, and time consuming to complete a flight test program depending on the number and type of required tests. Wind tunnel testing results in fairly accurate values of these coefficients, but it is considered a complicated and a time consuming method because several scale, interference and dynamic effects must be taken into account during refining its results. The CFD can also be used to calculate force and moment characteristics on aircraft. This method provides less precision than the wind tunnel testing and takes lots of CPU time particularly if estimates of the coefficients are desired over a range of flight conditions.

Nowadays, several software packages exist that allow one to obtain rapid, economical and reasonably good estimation of aerodynamic stability and control characteristics. Among these software packages are LinAir, AVL, Tornado, Edge Euler, XFLR5 and Digital DATCOM. For the purpose of obtaining mathematical model for our UAV in this paper, Digital DATCOM is used. Digital DATCOM software, developed by U.S. Air Force, has been widely used for preliminary estimation of an aircraft's aerodynamic coefficients and its stability derivatives. Given an aircraft configuration declared in an ASCI text file, DATCOM will easily provide the estimation of the aircraft's aerodynamic coefficients and its stability derivatives for given atmospheric condition.

It is well known that to start control system design, an open-loop plant model is required. Stability and control coefficients and derivatives resulted from Digital DATCOM can be used to calculate the coefficients of state-space matrices. The resulting linear model can then be used for control design tasks. This paper aims to provide the step by step procedure for modeling small UAVs starting from determination of the aircraft stability and control derivatives till creating the open-loop plant model. A remote controlled UAV called "Sky Raider Mach 1" has been purchased and assembled to demonstrate this procedure.

The paper is organized as follows: Section II is devoted to setting up inputs to Digital DATCOM software. In Section III, a brief description on DATCOM software will be given. Section IV is devoted to import DATCOM outputs files into MATLAB for analysis and for further use in modeling flight vehicle dynamics and control system design. Finally, this paper concludes with a brief summary in Section V. 


\section{SETTING UP INPUTS TO DIGITAL DATCOM}

USAF Stability and Control Digital DATCOM is a program available to Langley Research Center users for computing static and dynamic stability derivatives as well as high lift and control power coefficients. The inputs to Digital Datcom define the flight conditions, the reference dimensions, the basic configuration geometry for conventional configurations; i.e. defining the body, wing and tail surfaces and their relative locations, and also specify additional configuration definition; such as engines, flaps, control tabs, ground effects or twin vertical panels [1].

\section{Physical Measurements and Mass Properties}

For the purpose of this paper, a remote controlled UAV called "Sky Raider Mach $1 "$, shown in Fig. 1, is used. It is a highwing tricycle-gear radio-controlled airplane. It is constructed of wood, foam, composites, and metal. It is powered by an O.S. 0.46-cubic-inch displacement 2stroke nitro engine which drives a $11 \times 8$ propeller from APC. Table 1: describes physical specifications of the Sky Raider Mach 1.

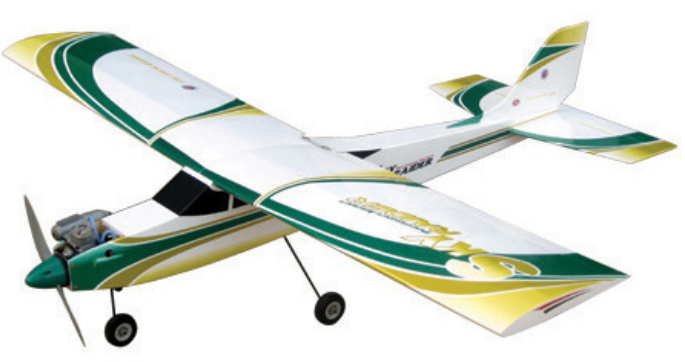

Fig. 1: Sky Raider model picture.

Table 1: Sky Raider physical dimensions.

\begin{tabular}{|l|c|c|l|c|c|}
\hline \multicolumn{1}{|c|}{ Name } & Symbol & Value & \multicolumn{1}{c|}{ Name } & Symbol & Value \\
\hline Wing Reference Area & $S_{W}$ & $0.468 \mathrm{~m}^{2}$ & Vertical Tail Area & $S_{V}$ & $0.0295 \mathrm{~m}^{2}$ \\
\hline Wing Span & $b_{W}$ & $1.66 \mathrm{~m}$ & Vert. Tail Span & $b_{V}$ & $0.175 \mathrm{~m}$ \\
\hline Wing Root Chord & $C_{R W}$ & $0.285 \mathrm{~m}$ & Vert. Tail Root Chord & $C_{R V}$ & $0.297 \mathrm{~m}$ \\
\hline Aspect Ratio & $A R=\frac{b_{W}}{C_{R W}}$ & 5.82 & Horiz. Tail Taper Ratio & $\lambda_{H}$ & 1.44 \\
\hline Horizontal Tail Area & $S_{H}$ & $0.109 \mathrm{~m}^{2}$ & Horiz. Tail Tip Chord & $C_{t H}$ & $0.15 \mathrm{~m}$ \\
\hline Horiz. Tail Span & $b_{H}$ & $0.595 \mathrm{~m}$ & Vertical Tail Taper Ratio & $\lambda_{V}$ & 3.19 \\
\hline Horiz. Tail Root Chord & $C_{R H}$ & $0.216 \mathrm{~m}$ & Vert. Tail Tip Chord & $C_{t V}$ & $0.093 \mathrm{~m}$ \\
\hline Aircraft Mass (Empty) & $W_{E}$ & $2.45 \mathrm{Kg}$ & & & \\
\hline
\end{tabular}

The aircraft employs a high-wing rectangular planform. Therefore the wing tip chord is equal to its root chord and consequently the taper ratio $(\lambda)$, the ratio of the tip to root chord, is unity. By observation the wing incidence, the angle the root chord makes with the fuselage reference line, is found to be 2 deg.

\section{Flight Conditions, Inertia Measurements and Centre of Gravity Calculations}

\section{Inertia calculations}

Having accurate moments of inertia is critical to ensure the accurate prediction of aircraft dynamics. Two methods exist to determine inertia. The first method uses 
direct calculation of a model's moments of inertia by consideration of the contributions made by individual parts. The second method uses determination of the moments of inertia by testing, which is much more practical and precise, hence this method was chosen. In determining the moments of inertia by test, the aircraft is hung from the ceiling and swung freely in the $X Y, X Z$, and $Y Z$ planes.

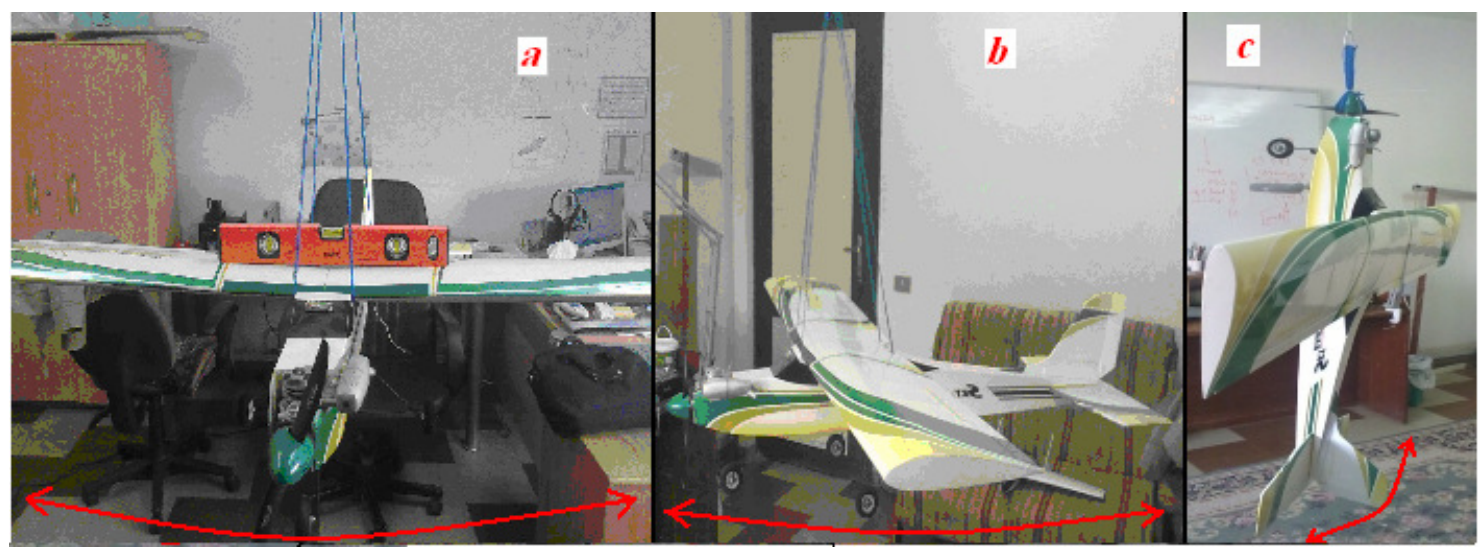

Fig. 2: Sky Raider hanging (a: $\left.I_{X X}, b: I_{Y Y}, c: I_{Z Z}\right)$.

Ref. [2] provides Eq. (1) for calculating the moment of inertia of a swinging model.

$$
I=\frac{\left[W_{M}+2 \omega\left(L_{\text {short }}+L_{\text {long }}\right)\right] \bar{Z}_{M+S}}{4 \pi^{2}} p_{M+S}^{2}-\frac{W_{M} \bar{Z}_{M}^{2}}{g}-\frac{2 \omega}{3 g}\left(L_{\text {short }}^{2}+L_{\text {long }}^{2}\right)
$$

where $W$ is the weight, $Z$ is the distance from pivot to center of gravity, $p$ is the period, and $g$ is the gravitational constant. $M$ and $S$ are subscripts designating either the model or the support. $L$ is the length of a chain. There are four chains; two "long" chains and two "short" chains, all having a weight per unit length $\omega$. The three variables $\bar{Z}_{M+S}, \bar{Z}_{M}$, and $p_{M+S}$ were measured for each of the three configurations and calculations were made. Four periods were timed during the swing tests, and the tests were repeated ten times. The moments of inertia calculated are shown in Table 2.

Table 2: Moments and products of inertia

\begin{tabular}{|c|c|}
\hline Axis of Oscillation & Inertia $\left(\mathrm{Kg} \cdot \mathrm{m}^{2}\right)$ \\
\hline$X$ & $\mathrm{I}_{\mathrm{XX}}=0.527$ \\
\hline $\mathrm{Y}$ & $\mathrm{I}_{\mathrm{YY}}=0.605$ \\
\hline$Z$ & $\mathrm{I}_{\mathrm{ZZ}}=0.496$ \\
\hline & $\mathrm{I}_{\mathrm{XZ}}=0$ \\
\hline
\end{tabular}

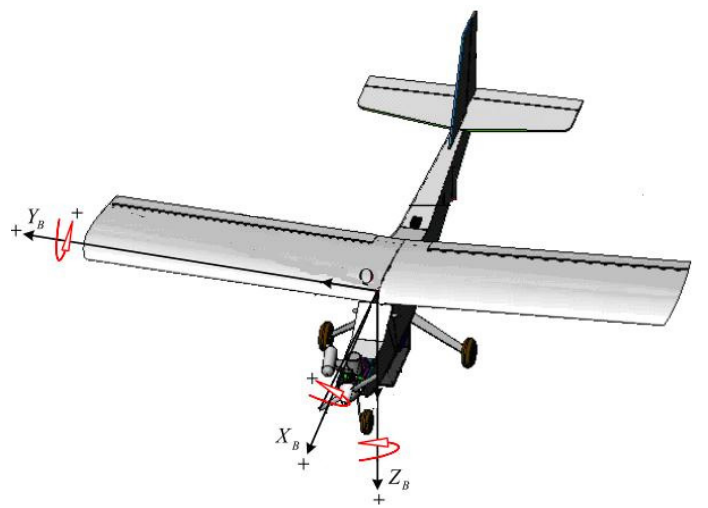

Fig. 3: Aircraft body axes.

\section{Center of gravity determination}

The CG was determined along all three axes. From the instruction manual of the 
aircraft, it is mentioned that the CG position along the $Y$ and $X$ axis is $80 \mathrm{~mm}$ behind the leading edge measured at where the wing meets the fuselage. The CG along the $Y$ axis was taken at the center. These two positions were temporarily marked on the fuselage. When the aircraft was hung for the moment of inertia swing, a laser beam was projected along the body to aid in finding the $Z$ axis location of the CG.

\section{Airfoil boundary detection}

To find out the airfoil employed by Sky Raider's wing, an interactive program for the design and analysis of subsonic isolated airfoils called "XFOIL" is used. Given the coordinates specifying the shape of a 2D airfoil, Reynolds and Mach numbers, XFOIL can calculate the pressure distribution on the airfoil and hence lift and drag characteristics. The program also allows inverse design; i.e. it will vary an airfoil shape to achieve the desired parameters. It is released under the GNU GPL [3]. The data obtained are then plotted and imported to another software called "Profili" [4]. This software assists in searching for the right airfoil, and analyzing the airfoil aerodynamic as follows: A side-view of the model wing tip airfoil in horizontal plane is pictured imported to Profili software which in turn automatically detects the new airfoil boundary and compared it with the saved airfoil data base. Profili showed that NASA airfoil 2415 matches the Sky Raider wing airfoil with coefficient of similitude of $79.1 \%$.

\section{Flight condition and Reynolds-number calculation}

In order to determine the correct Reynolds-number (Re) for the Sky Raider, an average flight speed, average wing chord, fluid density, and fluid viscosity were required. Our operational flight condition was assumed to be at sea-level; i.e. air density $\rho=1.225 \mathrm{~kg} / \mathrm{m} 3$ and air's viscosity $\mu=1.78 \mathrm{E}-5 \mathrm{~kg} / \mathrm{ms}$, with Mach number of 0.04408 (airspeed of $15 \mathrm{~m} / \mathrm{s}$ ) and the operational angle-of-attack ranges from -6 to 19 deg.

$$
\operatorname{Re}=\frac{\rho V l}{\mu}=\frac{1.225 \times 15 \times 0.285}{1.78 \times 10^{-5}}=2.942 E 5
$$

where $/$ is the wing mean aerodynamic chord.
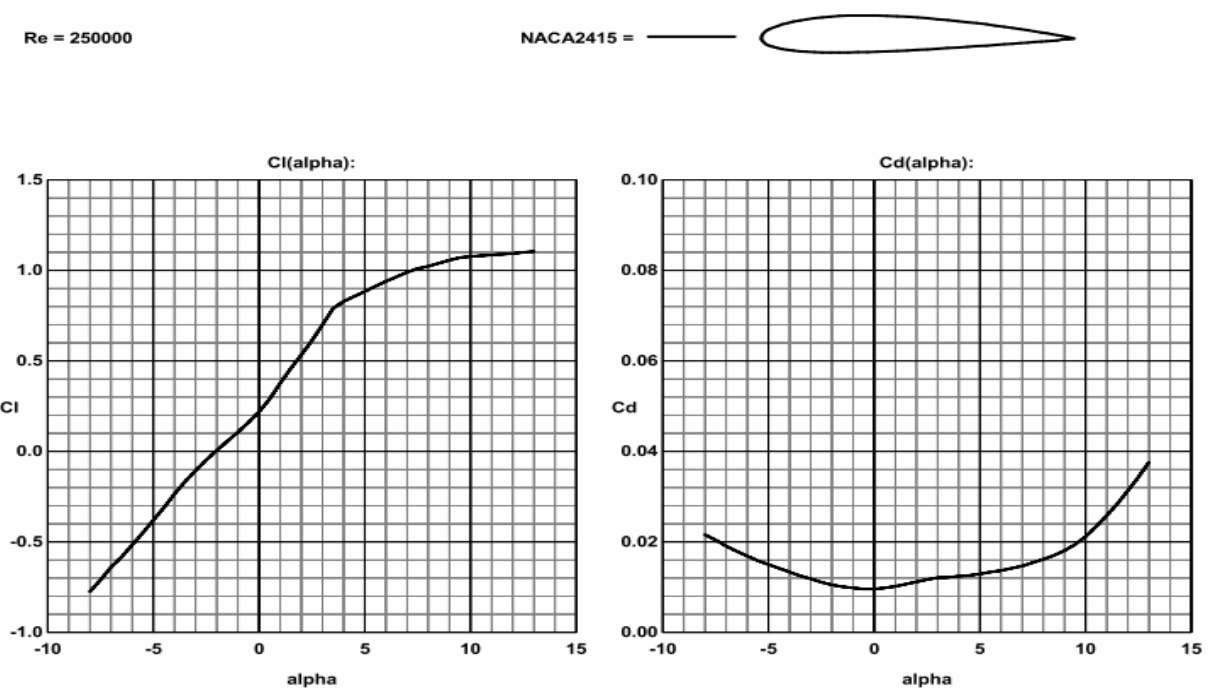

Fig. 4: Selected airfoil comparison. 


\section{Body Modeling}

The basic body geometry parameters required consist of the longitudinal coordinates with corresponding planform half widths, peripheries, and/or cross-sectional areas. A reference plane defined from the center of the propeller to the end of the fuselage (with the aircraft in a level flight orientation) was established. The fuselage was then sliced at regular intervals, called Stations, and the cross sectional area was determined with respect to the distance from the front of the cowling $(X)$ and the distance above (ZU ) and below (ZL) the reference plane.

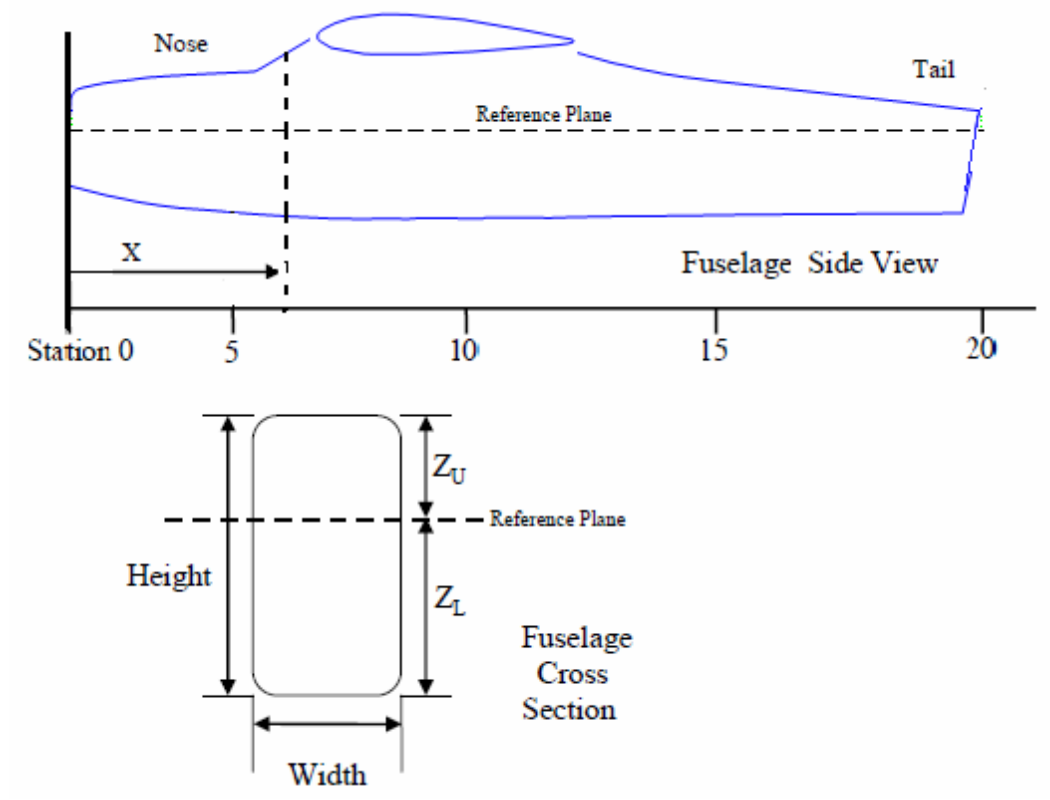

Fig. 5: Cross sectional area definitions.

Table 3: Sky Raider cross sectional dimensions.

\begin{tabular}{|c|c|c|c|c|c|c|c|c|c|}
\hline Station & $\boldsymbol{X}(\boldsymbol{m})$ & $\boldsymbol{S}\left(\boldsymbol{m}^{2}\right)$ & $\boldsymbol{Z}_{\boldsymbol{U}}(\boldsymbol{m})$ & $\boldsymbol{Z}_{\boldsymbol{L}}(\boldsymbol{m})$ & Station & $\boldsymbol{X}(\boldsymbol{m})$ & $\boldsymbol{S}\left(\boldsymbol{m}^{2}\right)$ & $\boldsymbol{Z}_{\boldsymbol{U}}(\boldsymbol{m})$ & $\boldsymbol{Z}_{\boldsymbol{L}}(\boldsymbol{m})$ \\
\hline 0 & 0 & 0.002 & 0.019 & -0.019 & 10 & 0.352 & 0.016 & 0.123 & -0.058 \\
\hline 1 & 0.006 & 0.003 & 0.028 & -0.031 & 11 & 0.393 & 0.015 & 0.12 & -0.056 \\
\hline 2 & 0.031 & 0.005 & 0.036 & -0.038 & 12 & 0.444 & 0.014 & 0.112 & -0.054 \\
\hline 3 & 0.083 & 0.008 & 0.045 & -0.047 & 13 & 0.543 & 0.012 & 0.088 & -0.05 \\
\hline 4 & 0.208 & 0.010 & 0.057 & -0.056 & 14 & 0.545 & 0.012 & 0.094 & -0.05 \\
\hline 5 & 0.279 & 0.015 & 0.102 & -0.059 & 15 & 0.621 & 0.010 & 0.076 & -0.047 \\
\hline 6 & 0.28 & 0.015 & 0.106 & -0.059 & 16 & 0.72 & 0.008 & 0.062 & -0.042 \\
\hline 7 & 0.281 & 0.015 & 0.107 & -0.059 & 17 & 1.161 & 0.001 & 0.027 & -0.025 \\
\hline 8 & 0.289 & 0.016 & 0.112 & -0.059 & 18 & 1.206 & 0.001 & 0.024 & -0.023 \\
\hline 9 & 0.311 & 0.016 & 0.12 & -0.059 & 19 & 1.214 & 0.000 & 0.023 & 0 \\
\hline
\end{tabular}




\section{Engine and Propeller Model}

Our Sky Raider UAV is powered by an O.S. 0.46cubic-inch displacement 2-stroke nitro engine (OS max. 46 AX) which drives a fixed pitch $11 \times 8$ propeller from APC. Modeling an engine with a propeller is started with a simplified modeling of the thrust generated assuming that the engine is overpowered providing enough torque to the propeller. Hence the thrust delivered by the propeller is only dependent on the aerodynamics of the propeller.

\begin{tabular}{|c|}
\hline Table 4: OS max 46 AX \\
Specifications \\
\hline Displacement: $7.5 \mathrm{cc}$ \\
Bore: $22.0 \mathrm{~mm}$ \\
Stroke: $19.6 \mathrm{~mm}$ \\
Practical rpm: $2,000-17,000$ \\
Output: $1.63 \mathrm{hp} @ 16,000 \mathrm{rpm}$ \\
Weight: $17.2 \mathrm{oz}(489 \mathrm{~g})$ with muffler \\
$13.2 \mathrm{oz}(375 \mathrm{~g})$ w/out muffler \\
\hline
\end{tabular}

The thrust force varies according to the rotating speed of the propeller $(\Omega)$, which can be computed using the following relation:

$$
T=\frac{4}{\pi^{2} \rho \Omega^{2} R_{p}^{4} C_{T}}
$$

where, $R_{p}$ is propeller radius in $[\mathrm{m}], \Omega$ is the rotational speed in $[\mathrm{rad} / \mathrm{s}], C_{T}$ is the thrust coefficient.

Also the actual maximum static thrust can be calculated using the momentum theory approach which does not include any propeller blade drag or any losses at the tips of the propeller blades as follows [5]:

$T=P^{2 / 3}(2 \rho A)^{1 / 3}$

The area of the propeller disc is $A=\pi \frac{D^{2}}{4}=0.06131 \mathrm{~m}^{2}$

where $D$ is the propeller diameter. Using Eq. (4), the estimated maximum static thrust is equal to $60.558 \mathrm{~N}$

We estimated the actual static force to be $90 \%$ of the maximum static thrust; i.e. 54.5 $N$. In addition to the thrust calculation, an experiment was conducted to identify the actual thrust at different rotating speeds. A spring scale was used to measure the static thrust along a set of throttle commands that results in different rotating speeds as shown in Fig. 6. A correction to the calculated static thrust was done by comparing with the actual thrust measurement by taking into account the reduced mass flow rate over the downstream region due to the fuselage effect.

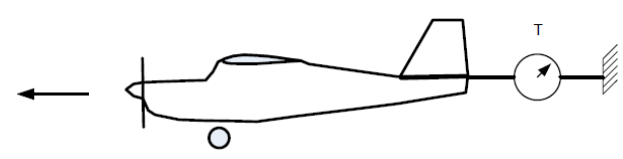

Fig. 6: Experimental setup for identifying static thrust force.
Table 5: Static thrust measurements at different RPM.

\begin{tabular}{|c|c|c|}
\hline Throttle position & Thrust (N) & RPM \\
\hline $25 \%$ & 5.3 & 2950 \\
\hline $50 \%$ & 12.1 & 6100 \\
\hline $75 \%$ & 22 & 8200 \\
\hline $100 \%$ & 30 & 11500 \\
\hline
\end{tabular}


The dynamic thrust calculation was held at cruse speed phase where throttle position is at $50 \%$. In general, the thrust force during flight depends not only on the rotational speed of the propeller but also on the forward flight speed (incoming wind speed). The forward flight speed reduces the generated thrust force by the propeller. The advance ratio $(J)$ is introduced to incorporate the effect of forward flight speed as follows [6]:

$$
\begin{aligned}
& J=\frac{V}{n D}=\frac{15(\mathrm{~m} / \mathrm{s})}{\frac{6000}{60} \times 0.2794}=0.536 \\
& C_{P}=\frac{P}{\rho n^{3} D^{5}}, \quad C_{T}=\frac{T}{\rho n^{2} D^{4}}, \quad \eta=J \frac{C_{T}}{C_{P}}
\end{aligned}
$$

where $n$ is the propeller's rotational speed in revolutions per unit of time, $C_{P}$ is the power coefficient, and $\eta$ is the efficiency coefficient.
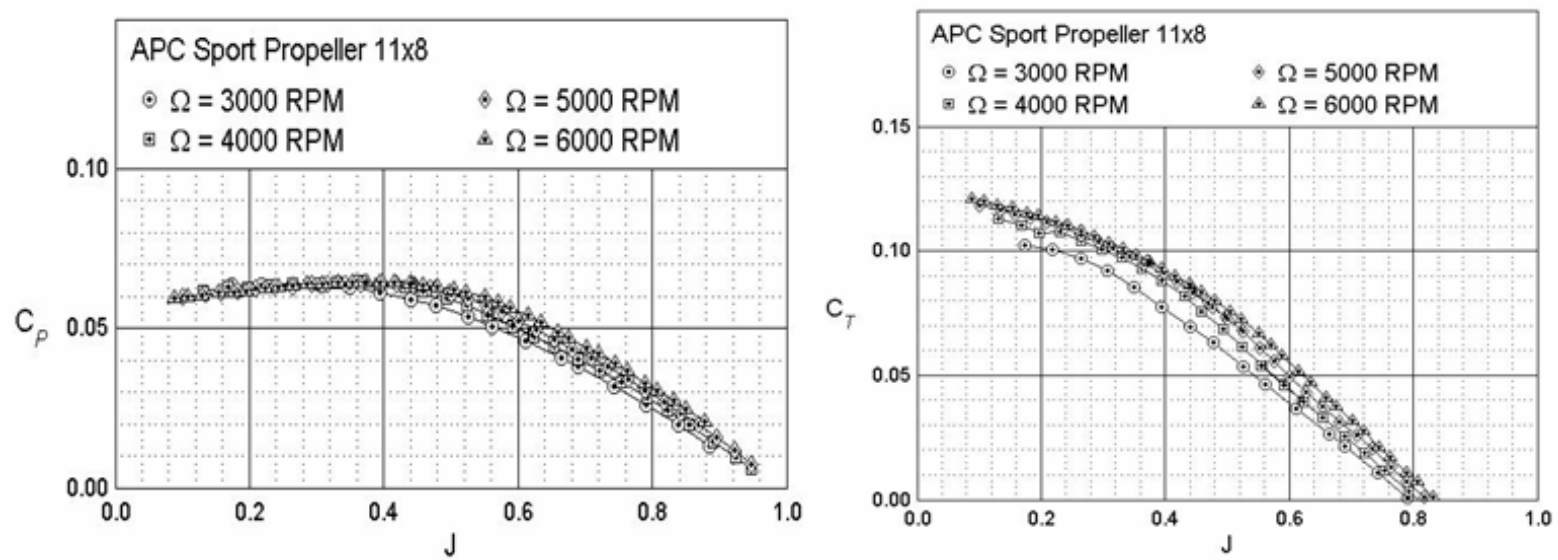

Fig. 7: Estimated APC 11x8 propeller performance data.

At the prescribed advance ratio, i.e. $J=0.536$, the thrust coefficient, the power coefficient and the propeller efficiency are found to be $0.071,0.063$ and $60 \%$, respectively.

\section{DIGITAL DATCOM SYSTEM OUTPUT}

After entering the input data described above, the digital DATCOM computed the static stability derivatives, the dynamic stability derivatives, and control derivatives for both the aileron and the elevator. The control derivatives associated with the rudder and the stability derivatives associated with the yawing moment are not computed from the DATCOM. These derivatives can be estimated using the method proposed in [7]. Among the digital DATCOM outputs, there is a file for the 3-D visualization of the aircraft model. The stability and control derivatives calculated from the digital DATCOM are summarized in Table 6, assuming no rudder deflection. 


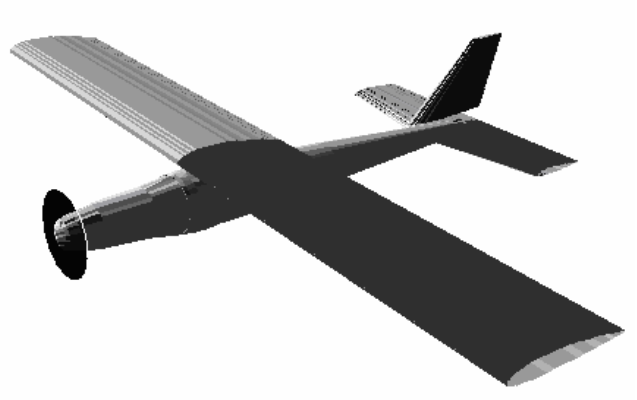

Fig. 8: Sky Raider 3-D visualization.

Table 6: Major digital DATCOM system output.

\begin{tabular}{|c|c|c|c|c|c|c|c|}
\hline$C_{D_{0}}$ & $C_{L_{0}}$ & $C_{L_{\alpha}}$ & $C_{L_{\dot{\alpha}}}$ & $C_{L_{q}}$ & $C_{y_{\beta}}$ & $C_{y_{p}}$ & $C_{y_{r}}$ \\
\hline 0.041 & 0.357 & 5.0 & 2.29 & 8.594 & -0.26 & 0.02622 & 0 \\
\hline$C_{m_{0}}$ & $C_{m_{\alpha}}$ & $C_{m_{\dot{\alpha}}}$ & $C_{m_{q}}$ & $C_{l_{\beta}}$ & $C_{l_{p}}$ & $C_{l_{r}}$ & $C_{n_{\beta}}$ \\
\hline-0.0399 & -1.514 & -6.6 & -14.44 & -0.0527 & 0.1731 & 0.08852 & 0.0324 \\
\hline$C_{n_{p}}$ & $C_{n_{r}}$ & $C_{D_{\delta_{e}}}$ & $C_{D_{\delta_{a}}}$ & $C_{D_{\delta_{r}}}$ & $C_{L_{\delta_{e}}}$ & $C_{y_{\delta_{a}}}$ & $C_{y_{\delta_{r}}}$ \\
\hline-0.03284 & -0.0448 & 0.011 & 0 & 0 & 0.304 & 0 & 0 \\
\hline$C_{m_{\delta_{e}}}$ & $C_{l_{\delta_{a}}}$ & $C_{l_{\delta_{r}}}$ & $C_{n_{\delta_{r}}}$ & $C_{n_{\delta_{a}}}$ & & & \\
\hline-0.8347 & 0.1585 & 0 & 0 & 0.02114 & & & \\
\hline
\end{tabular}

\section{CREATING AN OPEN-LOOP PLANT MODEL}

This section is devoted to importing DATCOM outputs files into MATLAB for obtaining the state variable representation of the aircraft equations of motion. The numerical values of the stability and control derivatives calculated from the digital DATCOM, Table 6, are substituted in the longitudinal and lateral directional derivatives given in Table 7.

Rearranging and collecting terms yield the longitudinal and lateral state space matrices of the state variable representation given by [8]:

$\dot{x}=A x+B u$

where $x$ is the state vector, $u$ is the control vector. 
Table 7: Summary of longitudinal and lateral directional derivatives.

\section{Longitudinal derivatives}

$$
\begin{aligned}
& X_{u}=\frac{-\left(C_{D_{u}}+2 C_{D_{0}}\right) Q S}{m u_{0}} \\
& X_{w}=\frac{-\left(C_{D_{\alpha}}-C_{L_{1}}\right) Q S}{m u_{0}} \\
& Z_{u}=\frac{-\left(C_{L_{\mu}}+2 C_{L_{0}}\right) Q S}{m u_{0}} \\
& Z_{w}=\frac{-\left(C_{L_{\alpha}}+C_{D_{0}}\right) Q S}{m u_{0}} \\
& Z_{\alpha}=u_{0} Z_{w} \\
& Z_{q}=C_{\mathrm{Z}_{\sigma}} \frac{\bar{c}}{2 u_{0}} Q S / m \\
& M_{u}=C_{m_{u}} \frac{(Q S \tilde{c})}{u_{0} I_{y}} \\
& M_{w}=C_{m_{\alpha}} \frac{(Q S \bar{c})}{u_{0} I_{y}} \\
& M_{\alpha}=u_{0} M_{w} \\
& M_{q}=C_{m_{q}} \frac{\bar{c}}{2 u_{0}}(Q S \bar{c}) / I_{y}
\end{aligned}
$$

Lateral directional derivatives

$$
\begin{array}{llll}
Y_{\beta}=\frac{Q S C_{y \beta}}{m}\left(\mathrm{ft} \mathrm{s}^{-2}\right) \quad N_{\beta}=\frac{Q S b C_{n \beta}}{I_{z}}\left(\mathrm{~s}^{-2}\right) \quad L_{\beta}=\frac{Q S b C_{l \beta}}{I_{x}}\left(\mathrm{~s}^{-2}\right) \\
Y_{p}=\frac{Q S b C_{y_{p}}}{2 m u_{0}} \quad(\mathrm{ft} / \mathrm{s}) \text { or }(\mathrm{m} / \mathrm{s}) \quad N_{p}=\frac{Q S b^{2} C_{n_{p}}}{2 I_{z} u_{0}} \quad\left(\mathrm{~s}^{-1}\right) \\
L_{p}=\frac{Q S b^{2} C_{l p}}{2 I_{x} u_{0}} \quad\left(\mathrm{~s}^{-1}\right) \\
Y_{r}=\frac{Q S b C_{y_{r}}}{2 m u_{0}} \quad(\mathrm{ft} / \mathrm{s}) \text { or }(\mathrm{m} / \mathrm{s}) & N_{r}=\frac{Q S b^{2} C_{n_{r}}}{2 I_{z} u_{0}} \quad\left(\mathrm{~s}^{-1}\right) \\
L_{r}=\frac{Q S b^{2} C_{l r}}{2 I_{x} u_{0}} & \left(\mathrm{~s}^{-1}\right) & \\
Y_{\delta a}=\frac{Q S C_{y_{\delta a}}}{m} & \left(\mathrm{ft} / \mathrm{s}^{2}\right) \text { or }\left(\mathrm{m} / \mathrm{s}^{2}\right) & Y_{\delta r}=\frac{Q S C_{y_{\delta r}}}{m} \quad\left(\mathrm{ft} / \mathrm{s}^{2}\right) \text { or }\left(\mathrm{m} / \mathrm{s}^{2}\right) \\
N_{\delta a}=\frac{Q S b C_{n \delta a}}{I_{z}} & \left(\mathrm{~s}^{-2}\right) & N_{\delta r}=\frac{Q S b C_{n \delta r}}{I_{z}} \quad\left(\mathrm{~s}^{-2}\right) \\
L_{\delta a}=\frac{Q S b C_{l_{\delta a}}}{I_{x}} & \left(\mathrm{~s}^{-2}\right) & L_{\delta r}=\frac{Q S b C_{l_{\delta r}}}{I_{x}} \quad\left(\mathrm{~s}^{-2}\right)
\end{array}
$$

The matrices $\boldsymbol{A}$ and $\boldsymbol{B}$ contain the aircraft's dimensional stability derivatives of both the longitudinal and lateral directional motions are given by:

$$
\begin{aligned}
A_{\text {long }} & =\left[\begin{array}{cccc}
X_{u} & X_{w} & 0 & -g \\
Z_{u} & Z_{w} & u_{0} & 0 \\
M_{u}+M_{\dot{w}} Z_{u} & M_{w}+M_{\dot{w}} Z_{w} & M_{q}+M_{\dot{w}} Z_{u_{0}} & 0 \\
0 & 0 & 1 & 0
\end{array}\right], B_{\text {long }}=\left[\begin{array}{cc}
X_{\delta_{e}} & X_{\delta_{r}} \\
Z_{\delta_{e}} & Z_{\delta_{r}} \\
M_{\delta_{e}}+M_{\dot{w}} Z_{\delta_{e}} & M_{\delta_{t}}+M_{\dot{w}} Z_{\delta_{r}} \\
0 & 0
\end{array}\right] \\
A_{\text {lat }} & =\left[\begin{array}{cccc}
\frac{Y_{\beta}}{u_{0}} & \frac{Y_{\beta}}{u_{0}} & -\left(1-\frac{Y_{r}}{u_{0}}\right) & \frac{g \cos \theta_{0}}{u_{0}} \\
L_{\beta} & L_{p} & L_{r} & 0 \\
N_{\beta} & N_{p} & N_{r} & 0 \\
0 & 1 & 0 & 0
\end{array}\right], \quad B_{\text {lat }}=\left[\begin{array}{cc}
0 & \frac{Y_{\delta_{r}}}{u_{0}} \\
L_{\delta_{a}} & L_{\delta_{r}} \\
N_{\delta_{a}} & N_{\delta_{r}} \\
0 & 0
\end{array}\right]
\end{aligned}
$$

These matrices are required for analysis and for further use in modeling flight vehicle dynamics and control system design. The homogeneous solution to Eq. (8) can be obtained by assuming a solution of the form:

$$
x=x_{r} e^{\bar{\lambda}_{r} t}
$$

Substituting Eq. (9) into Eq. (8) yields:

$[\bar{\lambda} I-A] x_{r}=0$

$\bar{\lambda}$ are the eigenvalues or the characteristic roots, $x_{r}$ are the eigenvectors, and $/$ is the identity matrix.

The eigenvalues and eigenvectors technique is used to analyzing the aircraft transient motion, because it enables to describe the dynamic stability. According to Table 8, the eigenvalues of longitudinal state matrix $\boldsymbol{A}_{\text {long }}$ reveal that there are two 
sets of complex poles: the low frequency lightly damped set, called long period (Phugoid) mode, and the high frequency well damped set called short period mode.

Table 8: Characteristics of longitudinal and lateral dynamic stability.

\begin{tabular}{|c|c|c|c|c|c|c|c|}
\hline \multicolumn{5}{|c|}{ For the longitudinal motion } & \multirow{5}{*}{$\begin{array}{l}\text { Eigenvalue } \\
-7.56 \pm 5.32 \mathrm{i} \\
-0.0528 \pm 0.67 \mathrm{i}\end{array}$} & \multirow{5}{*}{$\begin{array}{l}\text { Damping } \\
0.818 \\
0.0785\end{array}$} & \multirow{5}{*}{$\begin{array}{l}\text { Freq. } \\
(\mathrm{rad} / \mathrm{s}) \\
9.25 \\
0.673\end{array}$} \\
\hline & {$[-0.1443$} & 0.3765 & 0 & $-32.2]$ & & & \\
\hline \multirow{3}{*}{$A_{\text {long }}=$} & -1.2561 & -8.8683 & 49.21 & 0 & & & \\
\hline & 0.0497 & -0.6051 & -6.2132 & 0 & & & \\
\hline & 0 & 0 & 1.000 & 0 & & & \\
\hline \multicolumn{5}{|c|}{ For the lateral motion } & & & \\
\hline \multirow{4}{*}{$A_{\text {lat }}=$} & -0.45738 & 0 & -1.0 & 0.6533 & $-0.0891 \pm 3.06 \mathrm{i}$ & 0.0291 & 3.06 \\
\hline & -11.006 & -2.0043 & 1.0247 & 0 & -2.86 & 1.0 & 2.86 \\
\hline & 7.085 & -0.39839 & -0.54421 & 0 & 0.031 & -1.0 & 0.031 \\
\hline & 0 & 1.0 & 0 & 0 & & & \\
\hline
\end{tabular}

By the same manner, the eigenvalues of lateral state matrix $\boldsymbol{A}_{\text {lat }}$, shows there are two real poles and set of complex pole. The negative real pole, -2.86 , is corresponding to the roll subsidence mode, the positive real pole, 0.031 , is corresponding to the spiral mode, and the complex set is corresponding to the dutch-roll mode.

\section{CONCLUSION}

This paper represents a guide for modeling any UAV. As inputs for digital DATCOM; the physical measurements, CG, inertia, engine and propeller model, throttle and control surface calibrations were performed manually in the lab. The airfoil model was performed using special software packages such as Xfoil and Profili. In addition to digital DATCOM, MATLAB was used to simplify, accelerate the modeling process, and create the open-loop plant model. This open-loop plant model is required for analysis and for further use in modeling flight vehicle dynamics and control system design. The discussed procedures are applied to a small remote controlled UAV called "Sky Raider Mach 1". According to the low accuracy of digital DATCOM, there will be a mismatch between the aircraft physical model and the obtained mathematical model, therefore the flight control system to be designed for such mathematical model must be robust enough for this mismatch between models.

\section{REFERENCES}

[1] Hoak, D.E, Ellison, D.E., et al. "USAF Stability and Control Datcom." Air Forces Flight Dynamics Laboratory AFFDL-TR-79-3032. April 1979.

[2] Beer, Ferdinal P., Johnson, Russell E., Jr., "Vector Mechanics for Engineers", Statics, Fifth Edition, McGraw-Hill Book Company, New York, 1988.

[3] http://en.wikipedia.org/wiki/XFOIL

[4] http://www.profili2.com

[5] Online course on vehicle performance available: http://www.dept.aoe.vt.edu/lutze/ /AOE3104/thrustmodels.pdf 
[6] Brandt J. B. and M. S. Selig, "Propeller Performance Data at Low Reynolds Numbers," in 49th AIAA Aerospace Sciences Meeting, Orlando, Florida, 2011.

[7] Jung, D. and Tsiotras, P., "Modeling and Hardware-in-the-loop Simulation for a small Unmanned Aerial Vehicle", AIAA, Conference and Exhibit, California, 2007.

[8] Nelson, R. C.,"Flight Stability and Automatic Control", Second Edition, McGraw-Hill Book Company, New York, 1998. 